\title{
Transverse and longitudinal dynamics at RHIC
}

\author{
Pawel Staszel for the BRAHMS Collaboration \\ M. Smoluchowski Inst. of Physics, Jagiellonian University, ul. Reymonta 4, 30-059 Kraków, \\ Poland
}

DOI: http://dx.doi.org/10.5689/UA-PROC-2010-09/49

\begin{abstract}
The BRAHMS collaboration measured inclusive identified hadron spectra in the wide rapidity range $(0<y<3.5)$ for $\mathrm{Au}+\mathrm{Au}$ and $\mathrm{p}+\mathrm{p}$ collisions at $\sqrt{s_{N N}}=62.4$ and $\sqrt{s_{N N}}=$ $200 \mathrm{GeV}$. In this proceeding we present a set of observables, namely $d N / d y$ rapidity dependence of pions, protons and anti-protons, anti-particle to particle ratios and the centrality dependence of net-proton distribution measured in $\mathrm{Au}+\mathrm{Au}$ collision $\sqrt{s_{N N}}=200 \mathrm{GeV}$. We also present rapidity evolution of $p / \pi$ ratio measured in the the same reaction. These results are discussed as to what constrains they can put on the models that account for baryon transport both in the longitudinal and transverse direction.
\end{abstract}

\section{Introduction}

Relativistic heavy ion reactions provide a unique opportunity to form and study hot nuclear matter at energy densities in clear excess over the value $\left(\approx 1 \mathrm{GeV} / \mathrm{fm}^{3}\right)$ predicted from Lattice QCD needed to make the phase transition or cross over to matter dominated by degrees of freedom of quarks and gluons rather than of hadrons. The deposited energy is essential to understand the formation of this medium. Because baryon number is conserved the measured baryon distribution retains information about energy loss and allows the degree of nuclear stopping to be determined. Such measurements may also help distinguish between different mechanisms for transporting baryons to mid-rapidity.

The BRAHMS collaboration measurements of mesons [1] and net-protons [2] revealed the difference in the beam energy evolution of rapidity distribution for newly created matter in the collisions and the matter that inherits the baryon number of the colliding nuclei. The emerged picture contains information about the interplay between the baryon number transport and the distribution of the dissipated energy deposition. This interplay drives also the effective chemical properties of the produced media.

\section{Brahms detector}

The BRAHMS experiment operated on the RHIC Collider together with other experiments PHENIX, STAR and PHOBOS. BRAHMS detector setup consists of two movable, narrow spectrometer arms: the Mid-rapidity Spectrometer (MRS) which operates in large scattering angles and the Forward Spectrometer (FS) that operates at forward angles in the range from 2.3 to $15 \mathrm{deg}$ which corresponds to the pseudo-rapidity interval from 2 to 4 . Additionally, the setup consists of the global detectors used to determine the global features of the collision such 
as the overall particle multiplicity and collision vertex location. The MRS is composed of the single dipole magnet (D5) placed between two TPCs which are made use of tracking. Particle identification (PID) is based on Time-of-Flight (TOF) measurement in two scintillator walls positioned downstream of the D5 dipole magnet. In the FS, the momentum measurement is performed by the setup of 5 tracking devices and 3 dipole magnets. In the FS, for medium momenta particles the PID is provided by TOF measurement in TOF2 detector and the high momentum particles are identified using a Ring Imaging Cherenkov detector (RICH). Event though the spectometers have small apertures, the ability of the spectrometers rotations allowed for the coverage of the large rapidity and $p_{T}$ interval $(0-5 \mathrm{GeV} / \mathrm{c})$. In that sense BRAHMS was complementary to other experiments that measured at mid-rapidity but in much broader solid angles.

\section{Stopping and particle production}

Figure 1 (right) shows the rapidity density of net-protons which reflects the distribution of the excess baryons over anti-baryons after the collision. The vertical lines indicate locations of the baryons before collisions which are just beam rapidities for top AGS, SPS and RHIC energies. We can see that in the AGS energy regime, the baryons are stopped in the collision, which is reflected in the net-baryon distribution which is picked at the mid-rapidity. However,
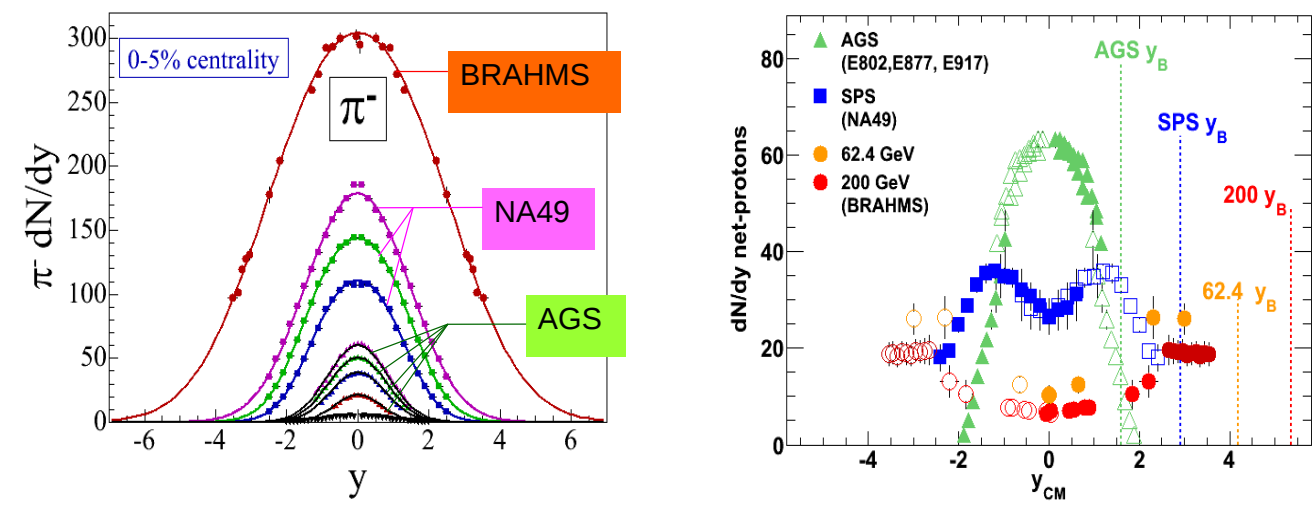

Figure 1: Right panel: evolution of net-proton distribution from lower to higher energies. Left panel: $\pi^{-} d N / d y$ rapidity distribution for AGS, SPS and RHIC energies. No significant change in shape from AGS to RHIC is observed.

going to higher energies the matter became more transparent, and the net-baryons start to concentrate at a constant distance from the initial beam rapidity. This lead to a small netbaryon density at mid-rapidity observed at RHIC energies. The increase of the transparency at higher energies can be seen from the figure showing average baryon rapidity loss $(\langle\delta y\rangle)$ versus the beam rapidity. The BRAHMS estimates for central Au+Au reactions at $\sqrt{s_{N N}}=62.4 \mathrm{GeV}$ and $\sqrt{s_{N N}}=200 \mathrm{GeV}$ show that the $\langle\delta y\rangle$ saturates above the SPS energy and do not show the linear increase known from measurements at the lower energies [3].

Figure 1 (left) shows the location of the matter that is produced in the collision. It can be seen from the plot that as the energy increases the amount of the produced matter also 
increases but the shape of the distribution does not significantly change from AGS to RHIC energies. The produced matter always concentrates at the mid-rapidity. These two tendencies at high energies lead to formation of very dense medium at mid-rapidity characterized with the low net-baryon content. We can calculate the average energy density in the transverse plane at mid-rapidity using the Bjorken formula [4]. For the maximum RHIC the obtained value exceeds $5 \mathrm{GeV} / \mathrm{fm}^{3}$ assuming the medium formation time of $1 \mathrm{fm} / c$.

Stopping and production influences the measured freeze-out anti-particle to particle ratios in the rapidity direction. In Figure 2 we plotted these ratios measured by BRAHMS in central $\mathrm{Au}+\mathrm{Au}$ collisions at $\sqrt{s_{N N}}=200 \mathrm{GeV}$. At $y \approx 0$ the $\bar{p} / p$ ratio is about 0.75 . The ratio decreases towards higher rapidities reaching value of about 0.3 at $y \approx 3$. Pions ratios are consistent with unity in the whole rapidity range, however, $K^{-} / K^{+}$ratios shows similar trend as this observed in $\bar{p} / p$ with less pronounced drop at high rapidity. At lower energy we observe similar picture
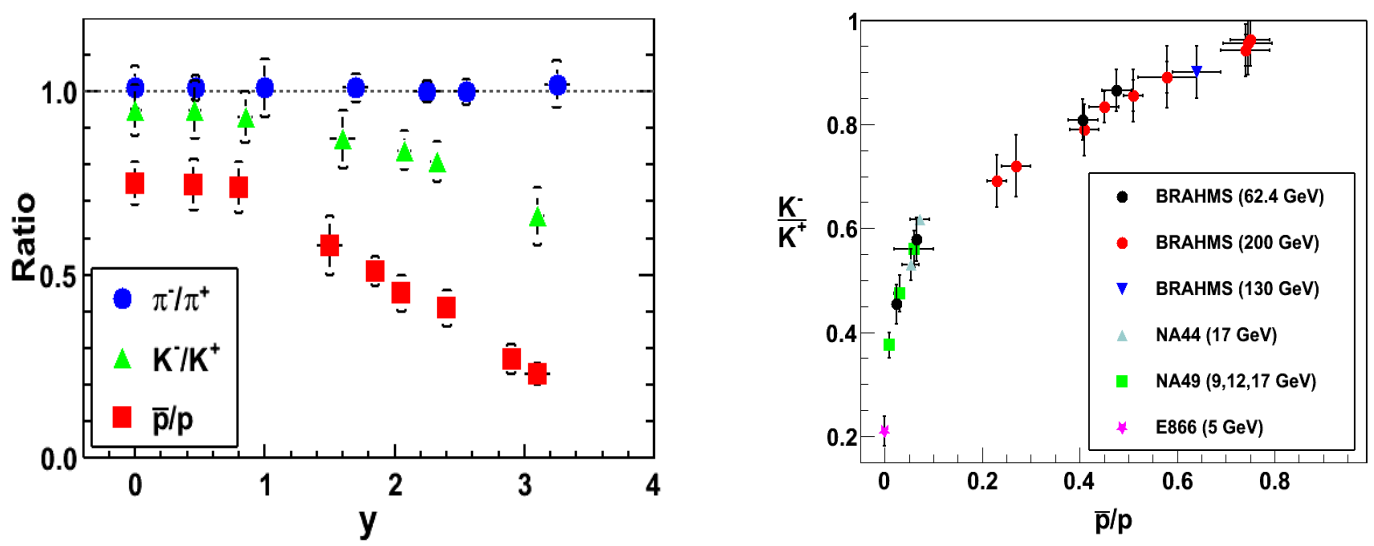

Figure 2: Left panel: Ratios of anti-particles to particles (pions, kaons and protons) as a function of rapidity for $\sqrt{s_{N N}}=200 \mathrm{GeV}$. Statistical and systematic errors are indicated. Right panel: Correlation between the BRAHMS kaon and proton ratios over 3 units of rapidity. Forward rapidity data at $\sqrt{s_{N N}}=62.4 \mathrm{GeV}$ overlap with mid-rapidity data from SPS

but the mid-rapidity proton and kaon ratios are lower and they drop faster toward forward rapidity [5]. Figure 2 (right) shows $K^{-} / K^{+}$ratios versus $\bar{p} / p$ ratios measured at the same rapidity intervals. We included data from $\mathrm{Au}+\mathrm{Au}$ at 200, 130 and $62 \mathrm{GeV}$ but also data for AGS and SPS energies. There is a striking correlation between the BRAHMS kaon and proton ratios over 3 units of rapidity. It is worth noting that the BRAHMS forward rapidity data measured at $\sqrt{s_{N N}}=62.4 \mathrm{GeV}$ overlap with the SPS points that were measured at much lower energy but at mid-rapidity. The data suggest that there is only one parameter that controls the relative particle abundances, namely, the $\bar{p} / p$ ratio. This can be also seen in $K^{+} / \pi^{+}$and $K^{-} / \pi^{-}$ratios measured at $\sqrt{s_{N N}}=62.4 \mathrm{GeV}$ and at forward rapidity which overlap with the similar ratios measured by NA49 in the range where the produced media at RHIC and SPS are described by the same value of $\bar{p} / p$ ratio. Dynamically the systems are, however different. The smaller inverse slope temperatures of $K^{+}$and $K^{-}$measured by BRAHMS as compare to SPS slopes in the $\bar{p} / p$ overlap region suggest that the larger radial flow develops in the second case.

In [6], Broniowski and Biedroń performed a fit to BRAHMS Au+Au data obtained at 
$\sqrt{s_{N N}}=200 \mathrm{GeV}$ using single freeze-out model (SFM) that incorporates rapidity dependence of baryon and strangeness chemical potentials. They found that the ratio of the baryon to strange chemical potentials is approximately 4 and constant over the whole rapidity interval covered by the data. Using the SFM the authors were able to reproduce the behavior of the strange chemical potential assuming vanishing local net-strangeness of the produced medium.

Taking $\mu_{S} \approx 1 / 4 \mu_{B}$ one gets from statistical model that $K^{-} / K^{+} \approx(\bar{p} / p)^{1 / 4}$ and this relation can successfully describe the correlation between kaon and proton ratios. The relation $\mu_{S} \approx 1 / 4 \mu_{B}$ can be used to predict correlation between anti-Hyperon to Hyperon ratios and the $\bar{p} / p$ ratio. The predictions, $\bar{\Lambda} / \Lambda \approx(\bar{p} / p)^{3 / 4}, \bar{\Xi} / \Xi \approx(\bar{p} / p)^{1 / 2}$ and $\bar{\Omega} / \Omega \approx(\bar{p} / p)^{1 / 4}$, agree well with the ratios measured at mid-rapidity at the wide range of energy in STAR and NA49 experiments [7].

\section{Model comparison}

It was argued above that the baryon stopping determines density and chemical composition of the produced media in high energy A+A collisions. Standard mechanism used for description of baryon transport involves quark - diquark string excitations followed by string breaking [8, 9] In this case the baryon number is associated with valence quarks. However, this mechanism alone is not able to move net-baryon number over a large range of rapidity. Analysis of $\mathrm{p}+\mathrm{p}$ ISR

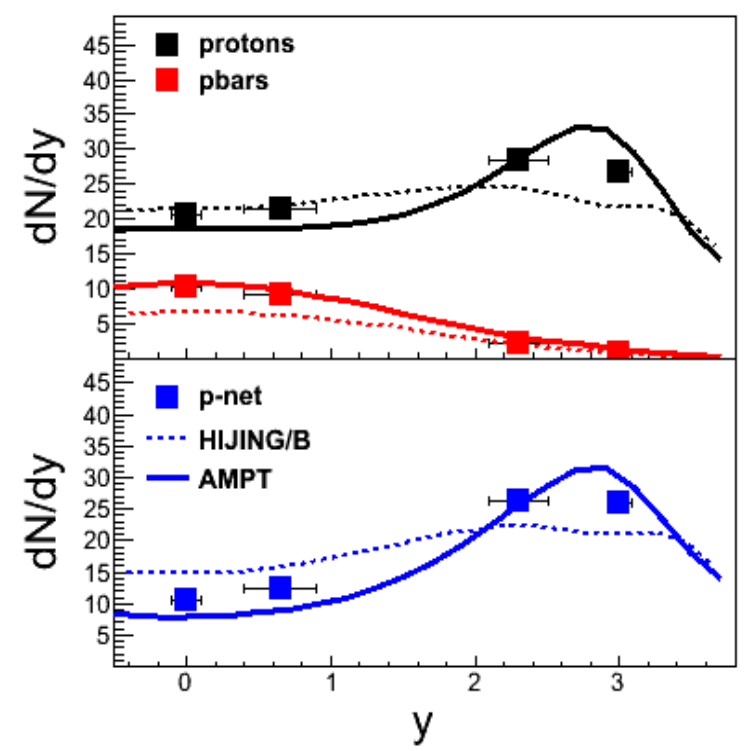

Figure 3: Top: rapidity densities of protons and anti-protons. Bottom: rapidity densities of net-protons. The statistical errors are smaller than the marker sizes [3].

data $[10,11]$ and HERA ${ }^{1}$ data [12] demonstrated that additional mechanisms with a slower $y$ dependence are needed to account for the data. One of such mechanism is baryon junction.

\footnotetext{
${ }^{1}$ The HERA experiment observed baryon asymmetry of $8 \%$ in $\gamma+p$ reactions at more that 7 units of rapidity from proton fragmentation.
} 
The concept of baryon junction was introduced in [13] and later developed in [14]. The baryon junction shows a weak dependency of baryon number transport on rapidity, thus event with small probability for creation, the baryon junction configuration can significantly contribute to baryon asymmetry observed in high energy reactions.

Figure 3 (top) shows proton and anti-proton rapidity distribution measured at central $\mathrm{Au}+\mathrm{Au}$ collisions at $\sqrt{s_{N N}}=62.4 \mathrm{GeV}$. The net-proton distribution is plotted in the bottom panel. For comparison we also plotted model prediction by AMPT [15] and HIJING/B [16] models. As it can be seen the AMPT model, that incorporates quark - diquark breaking mechanism provides overall good description but under estimates net-protons at mid-rapidity. HIJING/B model that incorporates baryon junction underestimates protons and net-protons at forward rapidity, however, this model can account for the large stopping. Note that the parameters used in HIJING/B calculations were tuned to describe data from the SPS.

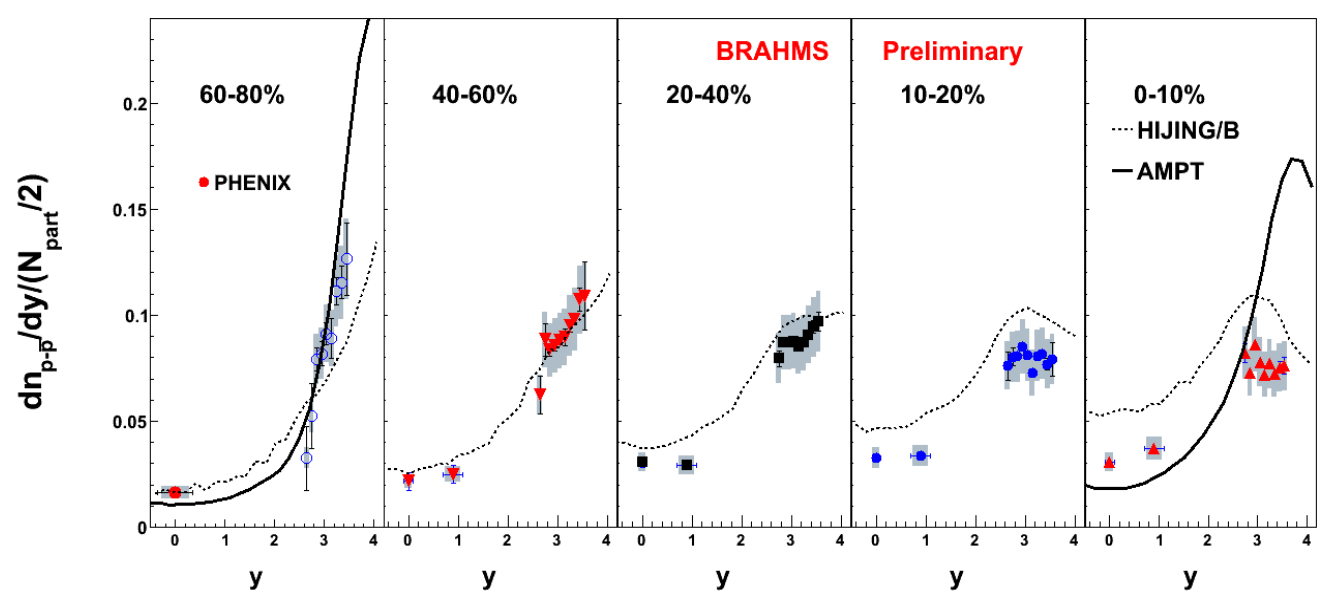

Figure 4: Net-proton yields in $\mathrm{Au}+\mathrm{Au}$ collisions at $\sqrt{s_{N N}}=200 \mathrm{GeV}$ normalized by $N_{\text {part }} / 2$ for different centrality samples. The single point at $\mathrm{y}=0$ in the $60-80 \%$ centrality sample is from PHENIX measurement [17]. No feed-down corrections for weak decays are applied.

The HIJING model without the junction (not shown) can describe the net-protons at $y \approx 0$ but the model underestimates the experimental $\langle\delta y\rangle$. It also significantly overestimates production of protons and anti-protons.

The net-proton distributions measured in $\mathrm{Au}+\mathrm{Au}$ at $200 \mathrm{GeV}$ for different centralities are presented in Figure 4. In order to remove dependency on $N_{\text {part }}$ and to focus on the shapes of distributions the data have been normalized by $N_{\text {part }} / 2$. The plotted lines representing model calculations show that AMPT provide a good description of peripheral $\mathrm{Au}+\mathrm{Au}$ data, this model, however can not account for data from central reactions. HIJING/B seems to reproduce the trend with centrality seen in data, however, it tends to overestimate net-protons for more central reactions.

The last figure (Fig. 5) presents $\eta$ dependence of the $p / \pi$ ratios measured in central $\mathrm{Au}+\mathrm{Au}$ collisions at $\sqrt{s_{N N}}=200 \mathrm{GeV}[18]$. Positive ratios $\left(p / \pi^{+}\right)$(top row) shows an increasing trend with increasing rapidity from $1.0\left(\eta \approx 0, p_{T}=3 \mathrm{GeV} / \mathrm{c}\right)$ to about $2.5\left(\eta \approx 3, p_{T}=3 \mathrm{GeV} / c\right)$. In opposite, $\bar{p} / \pi^{-}$(bottom row) decreases with increasing rapidity (from $\approx 1$ at $\eta \approx 0$ to 0.4 


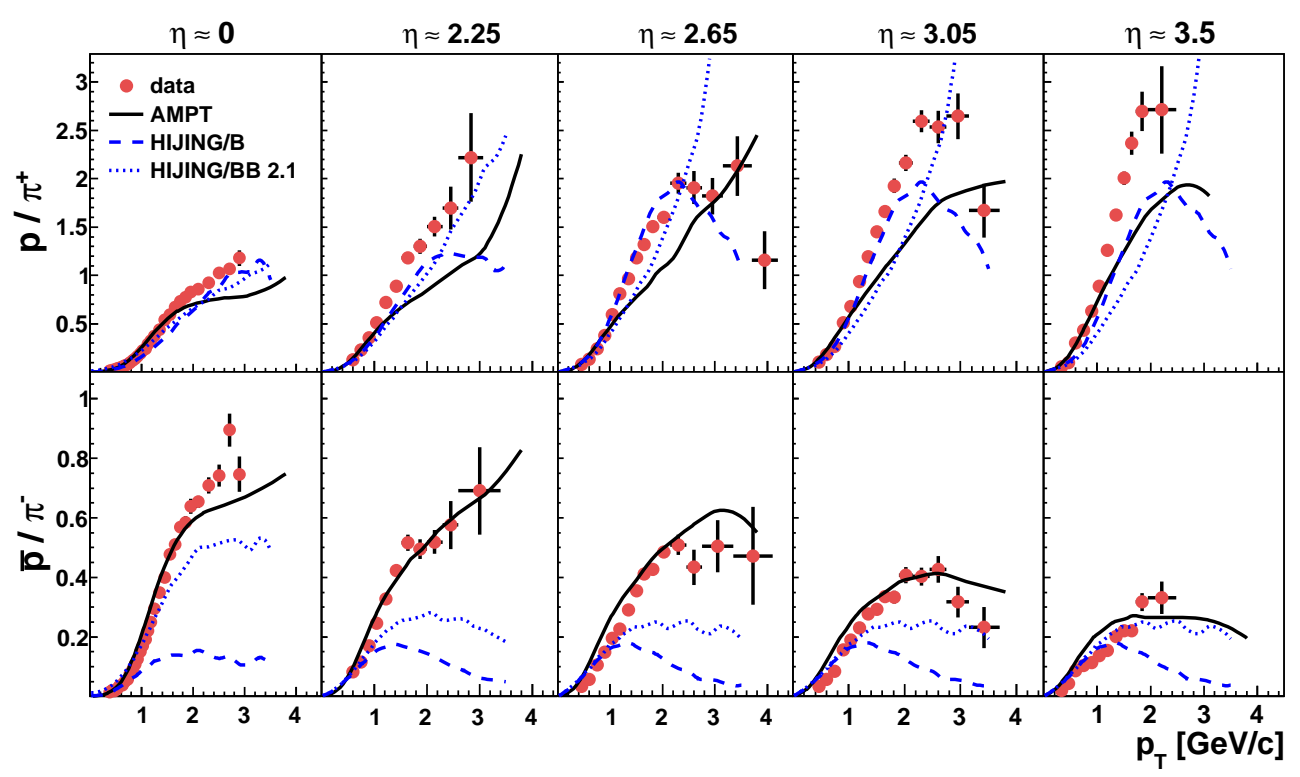

Figure 5: Forward region rapidity evolution of $p / \pi^{+}$and $\bar{p} / \pi^{-}$for $0-10 \%$ central $\mathrm{Au}+\mathrm{Au}$ reaction at $\sqrt{s_{N N}}=200 \mathrm{GeV}$ (solid points), and predictions from the AMPT model (solid line), HIJING/B model (dashed), and HIJING/B $\bar{B} 2.1$ (dotted line).

at $\eta \approx 3)$. Note a different vertical scales for positively and negatively charged species. In this figure we compare the data with three model calculation based on AMPT (solid line), HIJING/B

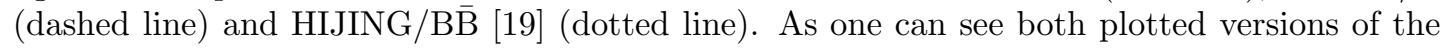
HIJING model are not able to describe the data which may indicate that the introduced baryon

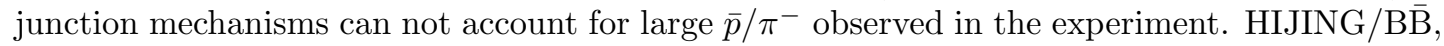
which incorporates modified baryon junction phenomenology to account for better description of hyperon $m_{T}$ spectra, does a somewhat better job in description of $p / \pi$ ratios, however, this version fails in the description of net-protons at $\sqrt{s_{N N}}=200 \mathrm{GeV}$. AMPT can quantitatively describe trends in rapidity evolution but fails in quantitative description, namely it under predicts $p / \pi^{+}$ratios. The description of $\bar{p} / \pi^{-}$ratio by AMPT is noticeably better than that provided by both version of the HIJING model. It may indicate that the initial state interaction is not able alone to account for the transverse dynamics observed in the data.

\section{Summary}

The BRAHMS experiment has provided measurements of baryon number transport in the $\mathrm{p}+\mathrm{p}$ and $\mathrm{Au}+\mathrm{Au}$ reactions at $\mathrm{RHIC}$ energies. The net-protons measured in $\mathrm{p}+\mathrm{p}$ [20] are consistent with quark - diquark breaking mechanism. The $\mathrm{Au}+\mathrm{Au}$ data suggest additional mechanisms for baryon transport (baryon junction, popcorn, di-quark breaking). To disentangle different scenarios for baryon stopping we studied both longitudinal and transverse dynamics of the baryon number transport. The presented analysis shows that none of the curent models, could 
simultaneously describe all available observables sensitive to baryon number transport like netprotons and $p / \pi$ ratios.

\section{References}

[1] I.G. Bearden, et al., BRAHMS Collaboration, Phys. Rev. Lett. 94 (2005) 162301.

[2] I.G. Bearden, et al., BRAHMS Collaboration, Phys. Rev. Lett. 93 (2004) 102301.

[3] I.C. Arsene, et al., BRAHMS Collaboration, Phys. Lett. B 677 (2009) 267.

[4] J. D. Bjorken, Phys. Rev. D 27 (1983) 140.

[5] A.I. Arsene, et al., BRAHMS Collaboration, Phys. Lett. B 687 (2010) 36.

[6] W. Broniowski, and B. Biedroń, Phys. Rev. C 75, 054905 (2007).

[7] C. Höhne for the NA49 Collaboration, Nucl. Phys. A 774 (2006) 35.

[8] S.E. Vance, M. Gyulassya and X-N. Wang, Nucl. Phys. A 638 (1998) 395c.

[9] A. Capella, J. Phys. G 23 (1997) 1979.

[10] G. Belletini et al., Nuovo Cimento A 42 (1977) 85.

[11] B. Z. Kopeliovich and A. Zacharov, Z. Physik C 446 (1999) 321.

[12] B. Z. Kopeliovich and B. Vogh, Phys. Lett. B 446 (1998) 241.

[13] G. C. Rossi and G. Veneziano, Nucl. Phys. B 123 (1977) 597.

[14] D. Kharzeev, Phys. Lett. B 378 (1996) 238.

[15] Z.W. Lin et al., Phys. Rev. C 72, 064901 (2005).

[16] S.E. Vance, M. Gyulassy, X-N. Wang, Phys. Lett. B 443 (1998) 45.

[17] S.S. Adler et al., Phys. Rev. C 69, (2004) 034909.

[18] I.C. Arsene, et al., BRAHMS Collaboration, Phys. Lett. B 684 (2010) 22.

[19] V. Topor Pop et al., Phys. Rev. C 70 (2004) 064906.

[20] F. Videbæk for the BRAHMS Collaboration, Nucl. Phys. A 830 (2009) 43c. 